\title{
¿Es leal el cliente de moda online? Claves de éxito para maximizar su lealtad en plata- formas de venta online
}

\author{
Adrián Castro-López, Javier Puente, Rodolfo Vázquez-Casielles \\ Recibido: 14 de Octubre de 2019 \\ Aceptado: 01 de Noviembre de 2019
}

https://doi.org/10.37610/dyo.v0i70.569

\section{Resumen}

En los últimos años, la moda se ha consolidado como uno de los sectores con mayor volumen de negocio a través de Internet. Sin embargo, algunos estudios indican que los clientes de moda no son fieles a una plataforma concreta. En el presente estudio, mediante la metodología de encuestas a una muestra de 405 clientes de plataforma de venta online en el sector de la moda, se analizan dos dimensiones de la experiencia online (calidad de servicio utilitaria y calidad de servicio hedónica), así como sus efectos directos e indirectos sobre la satisfacción y diversos factores de lealtad (recomendaciones, intención de recompra y tolerancia al precio). La conclusiones obtenidas indican que tanto la calidad utilitaria como la calidad hedónica tienen un impacto similar en la satisfacción, intenciones de recompra y tolerancia al precio. Sin embargo, la calidad hedónica tiene un efecto mayor sobre las recomendaciones (eWOM) de los consumidores que la calidad utilitaria (el doble). Así pues, es relevante potenciar prácticas de gestión de experiencias en las plataformas de venta online que impliquen una eficiente asignación de recursos para conseguir un balance equilibrado entre calidad utilitaria y calidad hedónica.

\section{Palabras clave}

Moda, calidad e-servicio, satisfacción, lealtad.

\section{Introducción}

La industria de la moda es una de las primeras creadas por el hombre, pese a lanzar al mercado productos básicamente tradicionales. Sin embargo, la rápida evolución de los mercados en los que opera, la corta duración del ciclo de vida de sus productos y su interdependencia con otras disciplinas como el arte, la cultura, la sociología, el marketing, la psicología del consumo, la logística o la ingeniería de organización, convierten al producto de moda en uno de los más difíciles de analizar por su evolución en el mercado de bienes de consumo (Blumer, 1969, del Olmo, 2010, Pedroni

\footnotetext{
$\square \quad$ Adrián Castro-López *

castroadrian@uniovi.es

https://orcid.org/0000-0001-8905-6251

Javier Puente **

jpuente@uniovi.es

https://orcid.org/0000-0001-7019-2202)

Rodolfo Vázquez-Casielles *

rvazquez@uniovi.es

https://orcid.org/0000-0002-6131-5183

* Dpto. de Administración de Empresas. Área de Comercial-

y Empresa. Universidad de Oviedo. Avda. del Cristo, s/n, 33006 Oviedo, Asturias (Spain).

** Dpto. de Administración de Empresas. Área de Organización de Empresas. Escuela Politécnica de Ingeniería de Gijón. Universidad de Oviedo. Campus de Viesques s/n, 33204 Gijón (Asturias)
}

y Pérez-Curiel, 2019). La industria de la moda se mueve a un ritmo vertiginoso, consumiendo ideas y tendencias con una velocidad sorprendente de modo que lo que hoy se considera en boga puede percibirse como obsoleto en pocas semanas. Así, resulta crucial para las empresas de este sector adaptarse con rapidez a los cambios en los gustos de los consumidores y comunicar y desarrollar acertadamente sus ideas y diseños al objeto de producir, ofrecer y distribuir sus productos en tiempo y forma a un grupo significativo de clientes (Moore, 2013) que podrían aumentar su lealtad e incluso ser embajadores de marca (Alfaro, 2013). Por ello, es muy importante establecer una estrategia de Marketing centrada en la optimización de recursos que maximice los resultados a nivel de volumen de ventas, satisfacción y lealtad del consumidor, aspectos cruciales para el éxito del comercio en este sector.

En la economía española, el sector de la moda es considerado estratégico al representar el 2,8\% de su Producto Interior Bruto (PIB) (Riaño, 2019). En concreto, en el ámbito productivo, la industria de la moda supuso en 2018 un 4,8\% de aportación al valor añadido bruto del sector manufacturero español. Por su parte, la distribución minorista y mayorista de moda en 2018 también alcanzó un $4,8 \%$ del valor añadido bruto de la distribución en España. Estas cifras denotan la relevancia del sector para el desarrollo económico del país.

También resulta relevante el análisis de la presencia del sector de moda a través de Internet. El último informe de la Comisión de los Mercados y de la Competencia sobre ecommerce (CNMC, 2019), indica que dicho sector se encuentra en cuarto lugar por volumen de negocio $(4,7 \%)$, tan solo 
precedido por las agencias de viajes y operadores turísticos $(16,5 \%)$, el transporte aéreo $(10,1 \%)$, y hoteles y alojamientos $(5,5 \%)$ (CNMC, 2019). Éste crecimiento ha supuesto que cada vez más empresas opten por este canal como medio de promoción y venta de sus productos (Taylor y Castello, 2017). El sector de la moda online se encuentra presente en diferentes tipos de plataformas: distribución de información, plataformas de venta y plataformas generadoras de opinión, como pueden ser los blogs (Martinez y Vázquez, 2011).

Nuestra investigación se centrará en el estudio de las plataformas de venta online de este sector, donde pueden diferenciarse cinco tipos principales: plataformas de marca y multimarca (p.e. Mango, Zara, Coach, El Corte Inglés, New Balance, Converse, Nike, JD), clubes de venta privada (p.e. Privalia, Veepee, Showroom Prive), Marketplaces (p.e. Amazon, Asos, Zalando, Sarenza, AliExpress), venta por catálogo online (Otto Group, La Redoutte, Spartoo) y subastas (p.e. Wallapop, Vinted).

Son numerosos los estudios que analizan la importancia de la calidad, tanto utilitaria (Cristóbal et al., 2007; Rares, 2014) como hedónica (Zhang et al., 2014; McLean, 2017). Se trata de dimensiones relevantes en la consecución de una adecuada percepción de calidad del e-servicio en una plataforma online. Sin embargo, no existen apenas estudios que analicen sus efectos directos e indirectos sobre la satisfacción del cliente y su lealtad a la hora de volver a adquirir un producto, recomendárselo a amigos y/o conocidos o incluso tener cierta tolerancia al precio al percibir el servicio como de calidad.

Así, el objetivo de nuestra investigación radica en la identificación de la relación entre la calidad utilitaria y hedónica como dimensiones de la calidad de e-servicio, y la satisfacción y lealtad del cliente online tras su experiencia en una plataforma de venta online en el sector textil y moda. Para ello, se desarrollará un modelo que incorpore los efectos directos e indirectos de la experiencia vinculada con la cali- dad de e-servicio en la satisfacción y la lealtad (intención de recompra, boca-oído (WOM) y tolerancia al precio), para analizar la importancia de cada una de estas variables y establecer las estrategias de Marketing online correspondientes para un mayor éxito empresarial.

Para llevar a cabo esta investigación, después de esta breve introducción, en el apartado 2 se analiza el estado del arte relativo a la calidad de e-servicio, su influencia sobre la satisfacción y los factores que explican la lealtad del cliente en plataformas de venta online. Posteriormente, en el apartado 3, se explica la metodología llevada a cabo para estimar el modelo propuesto. Por último, en el apartado 4, se describen las principales conclusiones derivadas del estudio.

\section{Estado del arte}

En este apartado se analiza la calidad de e-servicio y su influencia sobre la satisfacción, el boca-oído, la intención de recompra y la tolerancia al precio.

La calidad del e-servicio se entiende como la medida en la que un sitio web facilita la compra y entrega de forma eficiente y efectiva (Parasuraman et al., 2005) o desde un enfoque más amplio, la evaluación del cliente (en términos de aspectos hedónicos y utilitarios o funcionales) sobre la calidad del proceso y el resultado de la interacción con los canales online del proveedor del servicio. Son diversos los modelos que analizan tanto la calidad utilitaria, entendida como el valor derivado de completar los objetivos en la búsqueda de información y/o compra, como la calidad hedónica que hace alusión al valor derivado de la experiencia sensorial y emocional en la búsqueda de información y/o compra en una determinada plataforma de venta online. La Tabla 1 ilustra algunos de los modelos de evaluación de la calidad de e-servicio más relevantes y que han servido de soporte en investigaciones posteriores. 
Tabla 1 Modelos de evaluación de la calidad de e-servicio más relevantes.

\begin{tabular}{|c|c|c|c|c|}
\hline Autor & Dimensiones & Variables Resultado & Sector & Modelo \\
\hline Yoo y Donthu (2001) & $\begin{array}{l}\text { Fácil uso, diseño, capacidad de } \\
\text { respuesta, seguridad }\end{array}$ & Calidad e-servicio. & Librería. & SITEQUAL \\
\hline $\begin{array}{l}\text { Francis } \underset{\text { y }}{(2002)} \text { White } \\
\end{array}$ & $\begin{array}{l}\text { Portal web, sistema de transac- } \\
\text { ción, entrega, servicio al cliente, } \\
\text { seguridad }\end{array}$ & $\begin{array}{l}\text { Calidad web. } \\
\text { Intención compra }\end{array}$ & Internet. & PIRQUAL \\
\hline Loiacono et al. (2002) & $\begin{array}{l}\text { Usabilidad, facilidad de uso, } \\
\text { entretenimiento, relaciones com- } \\
\text { plementarias }\end{array}$ & Calidad e-servicio & Librería. & WEBQUAL \\
\hline $\begin{array}{l}\text { Wolfinbarger y Gilly } \\
\qquad(2003)\end{array}$ & $\begin{array}{l}\text { Diseño, fiabilidad, privacidad/se- } \\
\text { guridad, servicio al cliente }\end{array}$ & Calidad e-servicio & Detallista online. & eTailQ \\
\hline $\begin{array}{l}\text { Hernon } \underset{(2005)}{y} \text { Calvert } \\
\text { (200) }\end{array}$ & $\begin{array}{l}\text { Facilidad de uso, contenido, fia- } \\
\text { bilidad, fácil acceso, seguridad, } \\
\text { privacidad y confianza, conex- } \\
\text { ión, soporte, flexibilidad, person- } \\
\text { alización, diseño }\end{array}$ & Calidad e-servicio & Librería. & E-SERVQUAL \\
\hline \multirow[t]{2}{*}{$\begin{array}{l}\text { Parasuraman et al. } \\
\text { (2005) }\end{array}$} & $\begin{array}{l}\text { E-S-QUAL: Eficiencia, disponi- } \\
\text { bilidad, cumplimiento, privaci- } \\
\text { dad. }\end{array}$ & $\begin{array}{l}\text { Calidad e-servicio } \\
\text { Calidad en la recuper- } \\
\text { ación del servicio }\end{array}$ & Comercio B2C. & $\begin{array}{c}\text { E-S-QUAL } \\
\text { E-RecS-QUAL }\end{array}$ \\
\hline & $\begin{array}{l}\text { E-RecS-QUAL: Responsabili- } \\
\text { dad, compensación, contacto }\end{array}$ & & & \\
\hline Cristóbal et al. (2007) & $\begin{array}{c}\text { Servicio al cliente, seguridad, } \\
\text { diseño web, gestión del pedido }\end{array}$ & Calidad e-servicio & $\begin{array}{l}\text { Agencia de viajes } \\
\text { online. }\end{array}$ & PeQS \\
\hline $\begin{array}{c}\text { Bressolles y Durrieu } \\
\text { (2010) }\end{array}$ & $\begin{array}{l}\text { Facilidad de uso, información, } \\
\text { diseño, fiabilidad, seguridad/ } \\
\text { privacidad, interactividad/per- } \\
\text { sonalización }\end{array}$ & Calidad e-servicio & $\begin{array}{c}\text { Portales web de } \\
\text { vino }\end{array}$ & Netqual \\
\hline $\begin{array}{c}\text { Barrutia y Gilsanz } \\
\text { (2013) }\end{array}$ & $\begin{array}{l}\text { Recursos del cliente: Pericia del } \\
\text { entorno social, innovación } \\
\text { Recursos de la empresa: Calidad } \\
\text { de proceso, calidad de resultado. } \\
\text { Variables de control: Privacidad, } \\
\text { disfrute, equidad de pago }\end{array}$ & $\begin{array}{c}\text { Valor. } \\
\text { Satisfacción. } \\
\text { Intención de compor- } \\
\text { tamiento }\end{array}$ & $\begin{array}{l}\text { Servicios elec- } \\
\text { trónicos B2C }\end{array}$ & $\begin{array}{c}\text { Co-creación de } \\
\text { valor. }\end{array}$ \\
\hline $\begin{array}{l}\text { Zehira y Narcıkarab } \\
\text { (2016) }\end{array}$ & $\begin{array}{l}\text { Eficiencia, disponibilidad del } \\
\text { sistema, cumplimiento, compen- } \\
\text { sación y contacto }\end{array}$ & $\begin{array}{l}\text { Calidad e-servicio, } \\
\text { servicio post venta, } \\
\text { valor percibido, le- } \\
\text { altad }\end{array}$ & $\begin{array}{l}\text { Comercio mino- } \\
\text { rista online }\end{array}$ & e-Service quality \\
\hline Zhou et al. (2018) & $\begin{array}{l}\text { Percepción funcional, rendimien- } \\
\text { to, calidad de la plataforma e in- } \\
\text { teractividad, calidad de la infor- } \\
\text { mación y servicio postventa }\end{array}$ & $\begin{array}{l}\text { Calidad e-servicio, } \\
\text { satisfacción y lealtad }\end{array}$ & Telecom & e-Service quality \\
\hline Bleier et al. (2019) & $\begin{array}{l}\text { Capacidad de información (cog- } \\
\text { nitiva), entretenimiento (afecti- } \\
\text { va), presencia social, atractivo } \\
\text { sensorial }\end{array}$ & $\begin{array}{l}\text { Experiencia online y } \\
\text { compra del cliente }\end{array}$ & $\begin{array}{l}4 \text { empresas For- } \\
\text { tune } 1000 \text { de vari- } \\
\text { os sectores }\end{array}$ & $\begin{array}{l}\text { Experiencia on- } \\
\text { line del cliente }\end{array}$ \\
\hline
\end{tabular}


Los modelos de satisfacción cognitivos parten de la idea de que la satisfacción es una respuesta del consumidor que deriva de la evaluación comparada entre el resultado conseguido con un producto y un determinado estándar de referencia (Liljarder y Strandvik, 1997). Asimismo, numerosas investigaciones inciden en la importancia de la inclusión de las emociones en el ámbito del servicio, pues la satisfacción del cliente se centra principalmente en la experiencia previa al mismo (Grönroos, 2000) que al interactuar con factores ambientales y personales que rodean a la experiencia de compra, hacen que la comprensión de este tipo de respuestas afectivas-cognitivas sean básicas para la determinación de la satisfacción del cliente (Bigné y Andreu, 2004; Fernandes y Moreira, 2019).

En concreto, respecto a la venta a través de plataformas de venta online existen diferentes estudios, como el de Dziewanoska (2015), que establecen que una buena calidad del e-servicio incrementa la percepción de satisfacción de los usuarios, especialmente cuando la calidad utilitaria es importante para el cliente y cuando también lo es su experiencia sensorial o calidad hedónica en la plataforma de venta online. Por ello, se proponen dos hipótesis:

H1: La calidad utilitaria tiene influencia positiva en la satisfacción del cliente.

H2: La calidad hedónica tiene influencia positiva en la satisfacción del cliente.

La lealtad comienza en el momento en que el cliente está satisfecho con la compra de un bien o la prestación de un determinado servicio. En condiciones de libre competencia, un cliente leal siempre será un cliente satisfecho, pero un cliente satisfecho no siempre será un cliente leal. Un consumidor verdaderamente leal está comprometido con su empresa y es ese compromiso la causa de que vuelva a comprar en ella la próxima vez que necesite sus productos o servicios. En este caso, la lealtad del cliente online muestra paralelismos con el concepto de fidelidad de marca cuando un cliente visita una plataforma de venta online y/o realiza una compra en el mismo. Por este motivo, sería deseable maximizar la lealtad de los clientes online, aumentando el tiempo promedio empleado por el visitante en la plataforma online, así como las compras futuras (Dwi et al., 2018). Los clientes que estén satisfechos con su experiencia en la plataforma de venta online estarán más dispuestos a volver a utilizar o comprar en la misma, incluso pagando un recargo por los productos ofrecidos (tolerancia de precios). Del mismo modo, es probable que los clientes que perciben una alta calidad utilitaria y hedónica en su experiencia online estén más satisfechos (Holloway y Beatty 2008) y existe una mayor probabilidad de que éstos propaguen el eWOM positivo a sus amigos, familiares y/o conocidos (Yoo et al. 2013; Dost et al. 2019). Además, estarán dispuestos a hacer un mayor esfuerzo financiero (Homburg et al. 2005) y aumentar su intención de recompra (Martin et al., 2015).
Por el contrario, los clientes insatisfechos evitarán comprar en la plataforma nuevamente y darán mala prensa a la misma, causando un gran daño a su imagen (Yoo et al. 2015; Dost et al. 2019).

Por lo tanto, el estudio de la calidad e-servicio y la satisfacción del cliente juega un papel fundamental en la lealtad del cliente y, en consecuencia, en la supervivencia y el éxito de una organización (Kau y Lo 2006). Por ello, proponemos la siguiente hipótesis:

H3: La calidad utilitaria tiene influencia positiva sobre: a) eWOM, b) Intención de recompra y c) Tolerancia al precio.

H4: La calidad hedónica tiene influencia positiva sobre: a) eWOM, b) Intención de recompra y c) Tolerancia al precio.

H5: La satisfacción tiene influencia positiva sobre: a) eWOM, b) Intención de recompra y c) Tolerancia al precio.

\section{Metodología}

En este apartado se describe y evalúa la relación entre la calidad de e-servicio, la satisfacción, el eWOM, la intención de recompra y tolerancia al precio para usuarios habituales en plataformas de venta de moda en Internet.

Para ello, primeramente, se realizó una encuesta a clientes habituales mayores de 18 años que compran o buscan artículos de moda a través de Internet y que tienen su residencia establecida en España. La recogida de información se llevó a cabo mediante entrevistas personales y cuestionarios distribuidos por redes sociales como Facebook, LinkedIn y Twitter. El procedimiento de muestreo fue discrecional, teniendo en cuenta el tamaño de la muestra, del que se obtuvieron 405 encuestas válidas entre las 435 recibidas, con un error muestral de $\pm 2.42 \%$ para un nivel de confianza del $95 \%$. Entre los datos más relevantes obtenidos de la encuesta se puede destacar que el $71 \%$ de los clientes habituales de este tipo de plataformas son mujeres, frente al $29 \%$ de hombres. De estos usuarios, un $40 \%$ solo busca información y un $60 \%$ busca y compra artículos de moda en este tipo de plataformas. Además, entre los consumidores encuestados, el $34 \%$ ha realizado su primera compra/búsqueda en una tienda online de una marca concreta (p.e. Nike, Abercrombie and Fitch, Mango), el 31\% la ha realizado en un club de venta (p.e. Veepee, Showroom Prive), un 12\% en outfitters (p.e. Asos, Yoox), un 9\% en tienda de moda online por catálogo (p.e. La Redoutte, Venca) y un $14 \%$ en web de subastas y comercio colaborativo (p.e. eBay, Wallapop, Vinted). En cuanto al tipo de productos que adquieren los usuarios de este tipo de portales, la ropa de mujer representa un $30 \%$ de las compras, un $21 \%$ de los usuarios compran comple- 
mentos, un $17 \%$ ropa de hombre, un $18 \%$ zapatería, un $8 \%$ cosmética y perfumería y por último, un $6 \%$ de los usuarios encuestados adquieren ropa de niños. Por último, el 58\% de los usuarios gasta menos de 30 euros al mes, el 26\% entre 31-60 euros, el 12\% entre 61-100 euros, el 3\% entre 101-150 euros y un $1 \%$ gasta más de 150 euros al mes en artículos de moda en plataformas de venta online.

Una vez realizado el análisis descriptivo de la muestra de estudio, con el fin de evaluar la relación causal entre las diferentes variables indicadas, se utilizó un modelo de ecuaciones estructurales (Structural Equation Modeling, SEM) con ayuda del programa EQS 6.2 para Windows.

\subsection{Modelo de Ecuaciones Estructurales}

El modelo de ecuaciones estructurales (SEM) es una técnica que combina la regresión múltiple con el análisis factorial (Cupani, 2012; Hair et al., 2019) y que permite al investigador estimar una serie de ecuaciones de regresión múltiples distintas pero interrelacionadas mediante la especificación del modelo estructural. Para su desarrollo, en primer lugar, se utiliza la teoría, la experiencia previa y los objetivos de la investigación para determinar aquellas variables independientes que predicen cada variable dependiente del modelo de evaluación. A continuación, se determinan las relaciones entre las variables independientes y dependientes, incluso cuando una variable dependiente se convierte en independiente en otras relaciones (Hair, 1998). Una de las ventajas de este tipo de modelos radica en que permiten establecer el tipo y dirección de las relaciones que se espera encontrar entre las diversas variables contenidas en el modelo y poder contrastarlos con el modelo teórico propuesto, de ahí que sea uno de los análisis confirmatorios más utilizados (Ruiz et al., 2010).

De acuerdo con la Figura 1 y la Tabla 2, se puede establecer una relación directa, positiva y significativa entre las dimensiones de calidad utilitaria y hedónica con la satisfacción; otra relación entre la calidad utilitaria y la intención de recompra; otra entre la calidad hedónica y eWOM positivo; y por último, entre la satisfacción y la lealtad (eWOM positivo, intención de recompra y tolerancia de precios). Además, hay efectos de mediación de la satisfacción con respecto a la calidad y la lealtad ante un determinado servicio electrónico.

Figura 1 Modelo relación causal..

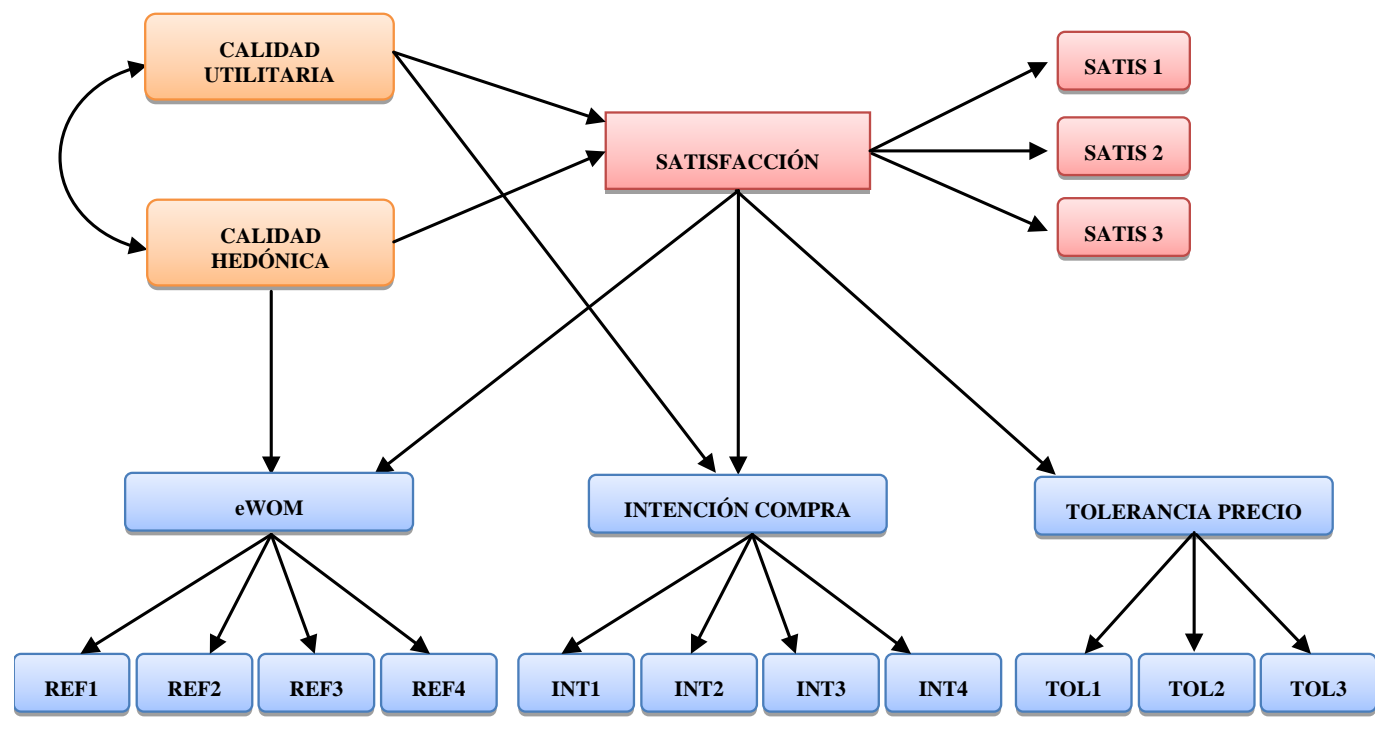

El análisis del modelo de ecuaciones estructurales descrito en la Figura 1 presenta buenos ajustes estadísticos (BBNN- 
Tabla 2 Relaciones entre calidad, satisfacción y lealtad.

\begin{tabular}{lc}
\hline \multicolumn{1}{c}{ Hipótesis } & Total muestra \\
\hline H1: Calidad utilitaria - Satisfacción & $0.42^{* *}$ \\
\hline H2: Calidad hedónica - Satisfacción & $0.44^{* *}$ \\
\hline H4a: Calidad utilitaria - eWOM & n.s. \\
\hline H4b: Calidad utilitaria - Intención de recompra & $0.07^{*}$ \\
\hline H4c: Calidad utilitaria - Tolerancia al precio & n.s. \\
\hline H5a: Calidad hedónica - eWOM & $0.23^{* *}$ \\
\hline H5b: Calidad hedónica - Intención de recompra & n.s. \\
\hline H5c: Calidad hedónica - Tolerancia al precio & n.s. \\
\hline H6a: Satisfacción - eWOM & $0.59^{* *}$ \\
\hline H6b: Satisfacción - Intención de recompra & $0.79^{* *}$ \\
\hline H6c: Satisfacción - Tolerancia al precio & $0.40^{* *}$ \\
\hline
\end{tabular}

Nota: ${ }^{*} \mathrm{p}<0,05 ; * * \mathrm{p}<0,01 ;$ n.s. $=$ no significativo
Una vez establecida la relación entre las variables, se estudiaron los diferentes efectos entre dichas variables. En nuestro caso, se utilizó la variable satisfacción como mediadora para determinar los efectos de las dos dimensiones de la calidad del e-servicio (utilitaria y hedónica) sobre la lealtad (eWOM positivo, intención de recompra y tolerancia de precios). El análisis se realizó de acuerdo con la evaluación de los efectos de mediación en un modelado de ecuaciones estructurales (Iacobucci, 2008). Para evaluar los efectos mediadores de la satisfacción, se verificaron los siguientes hechos (Iacobucci, 2008): (a) las variables independientes, es decir, las dimensiones de calidad utilitaria y hedónica, influyen en la variable mediadora (satisfacción); (b) la variable mediadora (satisfacción) afecta a variables dependientes como la lealtad. Además, cuando existe mediación total (efectos indirectos), el impacto de las variables independientes en las variables dependientes puede no ser significativo cuando se incluye la variable mediadora. En cambio, para el caso de existencia de mediación parcial (efectos directos e indirectos), cuando se incluye la relación entre las variables mediadoras y las variables dependientes, el efecto de las variables independientes en las variables dependientes sigue siendo significativo. Los resultados de esta investigación se muestran en la Tabla 3, donde se refleja el tipo de efecto (directo e indirecto) de las dimensiones de calidad utilitaria y calidad hedónica sobre la satisfacción y sobre los diferentes factores que explican la lealtad (eWOM, intención de recompra y tolerancia al precio). 
Tabla 3 Efectos directos e indirectos de la calidad de e-servicio sobre la lealtad.

\begin{tabular}{lcccc}
\hline \multirow{2}{*}{ Calidad e-servicio } & \multicolumn{4}{c}{ Efectos significativos } \\
\cline { 2 - 4 } & Satisfacción & eWOM & Intención de recompra & Tolerancia al precio \\
\hline \multirow{2}{*}{ Calidad Utilitaria } & Directo & Indirecto & Directo e Indirecto & Indirecto \\
\hline \multirow{2}{*}{ Calidad Hedónica } & Directo & Directo e Indirecto & Indirecto & Indirecto \\
\hline
\end{tabular}

En dicha Tabla 3 se observa como la calidad utilitaria y hedónica tienen efecto sobre la satisfacción e indirecto sobre la tolerancia al precio. Sin embargo, la calidad utilitaria tiene efecto indirecto sobre el eWOM y directo e indirecto sobre la intención de recompra. En el caso de la calidad hedónica, pasa lo contrario que con la utilitaria, pues tiene efecto indirecto sobre la intención de recompra y directo e indirecto sobre el eWOM.
Tabla 4 Resultados efectos directos e indirectos de la calidad de e-servicio sobre la lealtad.

\begin{tabular}{|c|c|c|c|c|c|c|c|c|c|c|}
\hline \multirow{2}{*}{$\begin{array}{l}\text { Dimensiones } \\
\text { calidad e-ser- } \\
\text { vicio }\end{array}$} & \multirow{2}{*}{$\begin{array}{l}\text { Satis- } \\
\text { facción }\end{array}$} & \multicolumn{3}{|c|}{ eWOM } & \multicolumn{3}{|c|}{ Intención de recompra } & \multicolumn{3}{|c|}{ Tolerancia al precio } \\
\hline & & Directo & $\begin{array}{l}\text { I n d i - } \\
\text { recto }\end{array}$ & Total & Directo & $\begin{array}{l}\text { I n d i - } \\
\text { recto }\end{array}$ & Total & Directo & $\begin{array}{l}\text { I n d i - } \\
\text { recto }\end{array}$ & Total \\
\hline $\begin{array}{l}\text { Calidad Utili- } \\
\text { taria }\end{array}$ & 0.42 & - & 0.25 & 0.25 & 0.07 & 0.33 & 0.40 & - & 0.17 & 0.17 \\
\hline $\begin{array}{l}\text { C a l i d a d } \\
\text { Hedónica }\end{array}$ & 0.44 & 0.23 & 0.26 & 0.49 & - & 0.35 & 0.35 & - & 0.18 & 0.18 \\
\hline
\end{tabular}

Por último, la Tabla 4 se resume los resultados numéricos relativos los efectos directos e indirectos y totales de la calidad de e-servicio en la satisfacción y la lealtad para toda la muestra analizada.
Así pues, las dimensiones de calidad utilitaria (0.42) y de calidad hedónica (0.44) tienen impacto directo positivo sobre la satisfacción. Tanto la calidad utilitaria como la calidad hedónica tienen un impacto similar sobre la satisfacción.

La calidad utilitaria no tiene un efecto significativo directo sobre el eWOM, pero si tiene un efecto indirecto a través de la satisfacción $(0.42 \times 0.59)=0.25$. Por tanto, el efecto total de la calidad utilitaria sobre el eWOM es de 0.25. Sin embargo, la calidad hedónica influye directamente $(0.23)$ e indirectamente a través de la satisfacción $(0.44 \times 0.59=$ 0.26) sobre el eWOM. El efecto total de la calidad hedónica sobre el eWOM es de $0.49(0.23+0.26)$. En definitiva, la calidad hedónica tiene un efecto mayor sobre el eWOM que la calidad utilitaria (el doble). Es muy importante crear emociones en el consumidor relacionadas con la navegación en la plataforma online para que ello origine recomendaciones y comunicación boca-oído.

La calidad utilitaria influye directamente $(0.07)$ e indirectamente a través de la satisfacción $(0.42 \times 0.79=0.33)$ en la intención de compra. El efecto total sobre la intención de recompra es de $0.40(0.07+0.33)$. La calidad hedónica no tiene un efecto significativo directo sobre la intención de recompra pero si tiene un efecto indirecto a través de la satisfacción $(0.44 \times 0.79=0.35)$. Por tanto, el efecto total de la calidad utilitaria sobre la intención de recompra es de 0.35 . Tanto la calidad utilitaria como la calidad hedónica tienen un impacto similar sobre la intención de recompra.

La calidad utilitaria no tiene un efecto significativo directo sobre la tolerancia al precio pero si tiene un efecto indirecto a través de la satisfacción $(0.42 \times 0.40=0.17)$. Por tanto, el efecto total de la calidad utilitaria sobre la tolerancia al precio es de 0.17 . La calidad hedónica tampoco tiene un efecto significativo directo sobre la tolerancia al precio pero si tiene un efecto indirecto a través de la satisfacción $(0.44$ x $0.40=$ 0.18). Por tanto, el efecto total de la calidad utilitaria sobre la tolerancia al precio es de 0.18. Tanto la calidad utilitaria como la calidad hedónica tienen un impacto similar sobre la tolerancia al precio.

\section{Conclusiones}

El presente estudio ha tratado de explicar la importancia de la evaluación de la experiencia de la calidad de e-servicio en plataformas de venta online en el sector textil y moda, fruto 
de una buena percepción de la calidad utilitaria y de la calidad hedónica en las mismas. En este estudio se analizaron los diferentes efectos de estas variables sobre aspectos relacionados con la lealtad como el eWOM, la intención de recompra y la tolerancia al precio utilizando como variable moderadora la satisfacción. Para llevarlo a cabo se analizaron 405 clientes habituales en plataformas de venta de productos de moda a través de Internet. Los resultados obtenidos muestran que las plataformas de venta online en el sector textil y moda deberán prestar atención a los efectos directos e indirectos de las dimensiones de calidad de e-servicio (utilitaria y hedónica) sobre variables resultado relacionadas con la lealtad (eWOM, intención de recompra y tolerancia al precio). Un análisis adecuado de dichos efectos permitiría a estas plataformas diseñar sus estrategias de marketing on-line para maximizar sus resultados a corto y largo plazo. El objetivo es adoptar estrategias comerciales que permitan una mayor satisfacción del cliente online para que vuelva a buscar información y comprar en la plataforma de venta online, hable positivamente de la misma a sus conocidos, amigos y familiares e incluso estén dispuestos a pagar un extracoste por adquirir el producto en la plataforma.

Tanto la calidad hedónica como la calidad utilitaria tienen un impacto muy similar sobre la satisfacción, la intención de recompra y la tolerancia al precio. Así pues, las plataformas de venta online deben continuar potenciando la calidad utilitaria para ser competitivas en el sector de textil y moda. Ello requiere inversiones en buen diseño web (atractivo, fácil de usar y con contenidos bien organizados), información de calidad (actualizada, de interés, en el idioma del consumidor y con tiempos de respuesta adecuados), garantía adecuada (facilidad de contacto empresa-cliente, garantía de sus productos, posibilidad de devolución y de identificación del pedido para poder trazarlo), oferta de productos y/o servicios de calidad (buena relación calidadprecio, promociones en la web, variedad de productos ofertados, precios competitivos), especial atención en la gestión en los pagos (posibilidad de crear cuentas con contraseña -registro-, identificación de los participantes en el sistema de pago), así como en la privacidad del servicio (garantía en la protección de datos y privacidad en la entrega) y en la confianza (posibilidad de cancelación del pedido, uso de certificado online y existencia de comentarios de otros consumidores) del consumidor en el portal web. Del mismo modo, las plataformas de venta on-line, para lograr satisfacción, intención de recompra y tolerancia al precio, han de ser innovadoras en calidad hedónica, proporcionando disfrutar al usuario en su navegación.

Sin embargo, la calidad hedónica tiene un efecto mayor sobre el eWOM que la calidad utilitaria (el doble). Para conseguir una buena calidad hedónica que origine recomendaciones y comunicación boca-oído, es necesario conseguir que el cliente se entretenga y disfrute navegando en la plata- forma de venta online, lo que implica conocer perfectamente los gustos del consumidor e incentivar que éste navegue en dicha plataforma. En definitiva, las plataformas de venta on-line pueden mostrar no sólo sus productos y diseños sino también otros contenidos relacionados con la moda como: próximos eventos, nuevas tendencias, comentarios y fotos de otros consumidores que llevan sus productos. Todo ello, por supuesto, utilizando las posibilidades que ofrecen las nuevas tecnología y las redes sociales en general, así como la realidad virtual en particular. Potenciar estas acciones, incidirá en conseguir que el consumidor sea un cliente habitual e incluso seguidor incondicional de la plataforma de venta online, siendo capaz de recomendar la plataforma entre sus amigos y conocidos.

Como limitaciones a este trabajo cabe destacar que sólo han sido analizadas las plataformas de venta cómo canal de venta online y sería interesante poder evaluar si el modelo se puede adaptar del mismo modo en otro tipo de plataformas, como pueden ser las generadoras de opinión. Además sería interesante poder analizar las experiencias del consumidor mediante un customer journey que integre tanto la búsqueda de información como la compra de productos de textil y moda a través de Internet, así como el efecto de la onmincanalidad en el sector de la moda, donde acciones como el webrooming y el showrooming permiten una mayor flexibilidad en el proceso de búsqueda y compra, traduciéndose en una mayor satisfacción y lealtad del cliente online con la plataforma. Por último, sería de gran relevancia conocer la influencia del concepto co-creación para aumentar la percepción de calidad de e-servicio, la experiencia y la lealtad del consumidor online. Dichas limitaciones serán objeto de estudio en una ampliación futura de esta línea de investigación.

\section{Referencias}

Alfaro, E. (2013). El ABC de la fanscination. Wolters Kluwer España. Madrid.

Barrutia, J.M., Echebarria, C., Paredes, M. (2013). Calidad de servicio electrónico y co-creación de valor: previsión de dos estudios empíricos. Cátedra Fundación Ramón Areces, Oviedo.

Bigné, J. E., \& Andreu, L. (2004). Modelo cognitivo-afectivo de la satisfacción en servicios de ocio y turismo. Cuadernos de Economía y Dirección de la Empresa, 21, pp.89-120.

Bleier, A., Harmeling, C.M., \& Palmatier, R.W. (2019). Creating effective online customer experiences. Journal of Marketing, 83(2), pp. 98-119.

Blumer, H. (1969). Fashion: From Class Differentiation to Collective Selection, The Sociological Quarterly, 10, pp. 275-291. 
Bressolles, G, Durrieu, F. (2010). A typology of online buyers for French wine web site based on electronic service quality dimensions. International Journal of Wine Business Research, 22(4), pp.335-348.

Cai, S., \& Jun, M. (2003). Internet users’ perceptions of online service quality: a comparison of online buyers and information searchers. Managing Service Quality: An International Journal, 13(6), pp. 504-519.

CNMC (2019) Informe sobre comercio electrónico del tercer trimestre de 2018. Madrid, España. https://www. cnmc.es/node/374283

Cristobal, E., Flavian, C., \& Guinaliu, M. (2007). Perceived e-service quality (PeSQ): measurement validation and effects on consumer satisfaction and web site loyalty. Managing Service Quality, 17(3), pp. 317-340.

Cristobal, E., Flavian, C., Guinaliu, M. (2007). Perceived e-service quality (PeSQ): measurement validation and effects on consumer satisfaction and web site loyalty. Managing Service Quality, 17(3), pp. 317-340.

Cupani, M. (2012). Análisis de Ecuaciones Estructurales: conceptos, etapas de desarrollo y un ejemplo de aplicación. Revista Tesis, 1, pp. 186-199.

Del Olmo, J.L. (2010). Marketing de la Moda. Ediciones Internacionales Universitarias S.A., Madrid.

Dost, F., Phieler, U., Haenlein, M., \& Libai, B. (2019). Seeding as part of the marketing mix: Word-of-Mouth program interactions for fast-moving consumer godos. Journal of Marketng, 83(2), 63-81.

Dwi, S., Ruhadi, R., Triyuni, N., \& Gundur, L. (2018). Online shopping loyalty: the role of quality, trust, value, and satisfaction. Advanced Science Letters, 2 4(1), pp. 735-738.

Dziewanowska, K. (2015). Dimensions of real and virtual consumer experiences. Faculty of Management Working Paper Series. University of Warsaw, 9(4), pp. 1-36.

Fernandes, T., \& Moreira, M. (2019). Consumer brand engagement, satisfaction and brand loyalty: a comparative study between functional and emotional brand relationships. Journal of Product \& Brand Management, 28(2), pp. 274-286.

Francis, J.E., \& White, L. (2002). PIRQUAL: A scale for measuring customer expectations and perceptions of quality. In Internet Retailing, Conference: Marketing Theory and Applications, 13, pp. 263-270.
Hair, J.F., Anderson, RE., Tatham, RL., \& William, CB. (1998). Multivariate Data Analysis. Prentice Hall, (5th ed.). Upper Saddle River, NJ: Prentice Hall.

Hair, J.F., Hult, T.M., Ringle, C.M., Sarstedt, M., Castillo-Apraiz, J., Cepeda-carrión, G., \& Roldán, J.L. (2019). Manual de Partial Least Squares Structural Equation Modeling (PLS-SEM). Sage Publishing (segunda edición).

Hernon, P., \& Calvert, P. (2005). E-service quality in libraries: exploring its features and dimensions. Library and Information Science Research, 27, pp. 377-404.

Holloway, BB., \& Beatty, SE. (2008). Satisfiers and dissatisfies in the online environment: a critical incident assessment. Journal of Service Research, 10(4), pp. 347-364.

Homburg C, Koschate N, \& Hoyer WD. (2005). Do satisfied customers really pay more? A study of the relationship between customer satisfaction and willingness to pay. Journal of Marketing, 69(2), pp. 84-96.

Iacobucci, D. (2008). Mediation analysis. Sage, London.

Kau A. \& Loh, EW. (2006). The effects of service recovery on consumer satisfaction: a comparison between complainants and non-complainants. Journal of Services Marketing, 20(2), pp. 101-111.

Liljander, V., \& Strandvik, T. (1997). Emotions in service satisfaction. International Journal of Service Industry Management, 8(2), pp.148-169.

Loiacono, E.T., Watson, R.T., \& Hoodhue, D.L. (2002). WEBQUAL: measure of website quality. Marketing Educators Conference: Marketing Theory and Applications, 13, pp. 432-437.

Martin, J., Mortimer, G., \& Andrews, L. (2015). Re-examining online customer experience to include purchase frequency and perceived risk. Journal of Retailing and Consumer Services, 25, pp. 81-95.

McLean, G. (2017). Investigating the online customer experience - A B2B perspective. Marketing Intelligence \& Planning, 35(5), pp. 657-672.

Moore, G. (2013). Promoción de moda, Editorial Gustavo Gilli S.L, México.

Parasuraman, A., Zeithaml, V., \& Malhotra, A. (2005). E-SQual. A multiple-item scale for assessing electronic service quality, Journal of Service Research, 7(10), pp. $1-21$. 
Pedroni, M. \& Pérez-Curiel, C. (2019). Investigación en Moda, Cambio e Industria. Revista Prisma Social, 24, pp. 1-9.

Rares, OD. (2014). Measuring perceived service quality offline vs. online: a new PeSQ conceptual model. Procedia Economics and Finance. 15, pp. 538-551.

RIAÑO, P. (2019). Informe Económico de la Moda en España. Moda.es. Madrid, España.

Ruiz, M., Pardo, A., \& San Martin, R. (2010). Modelos de ecuaciones estructurales. Papeles del Psicólogo. 31(1), pp. 34-45.

Taylor, CR., \& Costello, JP. (2017). What do we know about fashion advertising? A review of the literature and suggested research directions. Journal of Global Fashion Marketing, 8(1), pp. 1-20.

Verhoef, PC., Kannan, PK., \& Inman, JJ. (2015). From multichannel retailing to OmniChannel retailing: introduction to the special issue on multichannel retailing. Journal of Retailing, 91(2), pp. 174-181.

Wolfinbarger, M., \& Gilly, M.C. (2003). eTailQ: Dimensionalizing, measuring and predicting retail quality, Journal of Retailing, 79(3), pp. 183-198.
Yoo, B., \& Donthu, N. (2001). Developing a scale to measure the perceived quality of an Internet shopping site (SITEQUAL). Quarterly Journal of Electronic Commerce, 2(1), pp. 31-46.

Yoo, CW., Kim, YJ., \& Sanders, GL. (2015). The impact of interactivity of electronic word of mouth systems and e-quality on decision support in the context of the e-marketplace. Information \& Management, 52(4), pp. 496-505.

Zehira, C., \& Narcıkarab, E. (2016). E-Service Quality and E-Recovery Service Quality: Effects on Value Perceptions and Loyalty Intentions. 5th International Conference on Leadership, Technology, Innovation and Business Management. Procedia - Social and Behavioral Sciences, 229, pp. 427-443.

Zhang, H., Lu, Y., Gupta, S., \& Zhao, L. (2014). What motivates customers to participate in social commerce? The impact of technological environments and virtual customer experiences. Information \& Management, 51(8), pp. 1017-1030.

Zhou, R., Wang X., Shi, Y., Zhang, R., \& Zhang, L., Guo, H. (2018). Measuring e-service quality and its importance to customer satisfaction and loyalty: an empirical study in a telecom setting. Electronic Commerce Research, 19(3), pp. 477-499. 